\title{
In situ correction of field errors induced by temperature gradient in cryogenic undulators
}

\author{
Takashi Tanaka, ${ }^{1,2, *}$ Takamitsu Seike, ${ }^{2}$ Akihiro Kagamihata, ${ }^{2}$ Thomas Schmidt, ${ }^{3}$ Alexander Anghel, ${ }^{3}$ Mark Brügger, ${ }^{3}$ \\ Willy Bulgheroni, ${ }^{3}$ Bruno Jakob, ${ }^{3}$ and Hideo Kitamura ${ }^{1,2}$ \\ ${ }^{1}$ RIKEN/SPring-8, Koto 1-1-1, Mikazuki, Sayo, Hyogo 679-5148, Japan \\ ${ }^{2}$ JASRI/SPring-8, Koto 1-1-1, Mikazuki, Sayo, Hyogo 679-5198, Japan \\ ${ }^{3}$ Paul Scherrer Institute, 5232 Villigen PSI, Switzerland \\ (Received 10 September 2009; published 22 December 2009)
}

\begin{abstract}
A new technique of undulator field correction for cryogenic permanent magnet undulators (CPMUs) is proposed to correct the phase error induced by temperature gradient. This technique takes advantage of two important instruments: one is the in-vacuum self-aligned field analyzer with laser instrumentation system to precisely measure the distribution of the magnetic field generated by the permanent magnet arrays placed in vacuum, and the other is the differential adjuster to correct the local variation of the magnet gap. The details of the two instruments are described together with the method of how to analyze the field measurement data and deduce the gap variation along the undulator axis. The correction technique was applied to the CPMU with a length of $1.7 \mathrm{~m}$ and a magnetic period of $14 \mathrm{~mm}$. It was found that the phase error induced during the cooling process was attributable to local gap variations of around $30 \mu \mathrm{m}$, which were then corrected by the differential adjuster.
\end{abstract}

DOI: 10.1103/PhysRevSTAB.12.120702

PACS numbers: 07.85.Qe, 41.60.Ap

\section{INTRODUCTION}

The cryogenic permanent magnet undulator (CPMU), first proposed at SPring-8 [1], is an insertion device (ID) in which the permanent magnets (PMs) are cooled down to a cryogenic temperature to improve the magnetic performances in terms of the remanent field and coercivity. In particular, the coercivity is drastically improved and thus PM materials with a high remanent field, which cannot be applied to in-vacuum undulators (IVUs) at room temperature due to a low coercivity, are available to CPMUs. This is a great advantage over the conventional IVUs toward shortening the magnetic period and thus several facilities have already constructed prototype CPMUs [2-4].

Although CPMUs are realized by a slight modification of IVUs, i.e., by installing an additional equipment to cool down the PM arrays, we have several technical challenges to be overcome. Among them, the most important one is how to measure the magnetic field at a cryogenic temperature, and how to correct it if necessary. The ID field measurement is usually done by moving magnetic sensors such as Hall probes driven by an actuation stage with a high mechanical precision, supported by a long bench made of massive granite. This conventional method is, however, not applicable to CPMUs. We have to consider an alternative method to move precisely the magnetic sensor located in vacuum.

In 2007, a field measurement of the prototype CPMU with a magnetic length of $0.6 \mathrm{~m}$ was successfully carried out at SPring-8 [5], by means of inserting a Hall sensor

\footnotetext{
*ztanaka@ spring8.or.jp
}

cantilever into vacuum through dynamic reciprocating Teflon seals, and applying adaptive feedback of the Hall sensor position measured by a guiding laser with an optical axis coincident with the undulator axis. It was found that the peak field increased as the temperature decreased and reached maximum at $130 \mathrm{~K}$. It was also found that the magnetic performances in terms of the phase error and beam trajectory did not deteriorate by cooling down to the optimum temperature of $130 \mathrm{~K}$. This means that the magnetization vectors of individual PM pieces did not significantly change both in magnitude relative to average and in angle. From this point of view, the conventional undulator field correction, which is to be done at room temperature, is still effective even at a cryogenic temperature and is applicable to CPMUs as well as the other normal IDs.

In 2008, a field measurement of the CPMU with a length of $2 \mathrm{~m}$ constructed at ESRF was carried out by means of the specialized vacuum chamber dedicated to measurements at cryogenic temperatures [4]. It was found that the phase error increased by about $1^{\circ}$ at the optimum cryogenic temperature. This performance degradation was found to be attributable to a variation in the magnet gap along the undulator axis (gap variation) induced by temperature gradient, and can be an obstacle to the realization of longer CPMUs with less phase errors for the utilization of higher harmonics.

In order to compensate the temperature gradient and to correct the resultant phase error increase, a new method has been proposed at SPring-8, in which the gap variation is corrected by the mechanical adjustment of the out-vacuum shafts supporting the in-vacuum beam. What is important 
in this method is that the correction can be done at a cryogenic temperature without breaking the vacuum, i.e., an in situ field correction is possible.

The in situ correction method for CPMUs described above has been tested to check the feasibility using the new CPMU with a magnetic period of $14 \mathrm{~mm}$ and a magnetic length of $1.7 \mathrm{~m}$ (CPMU14), which has been constructed for installation in the Swiss Light Source storage ring in collaboration between PSI (Paul Scherrer Institute), SPring-8, and Hitachi Metals, Ltd. In this paper, the principle and results of the in situ correction applied to CPMU14 are described together with the details of the magnetic measurement system for CPMUs at a cryogenic temperature. Note that the details of CPMU14, including the cryogenics components and PM material, are out of the scope of this paper and will be described elsewhere.

\section{PRINCIPLE OF CORRECTION}

Let us assume that temperature gradient exists along the PM arrays of IDs. In conventional IDs, this gives rises to a variation in the remanent field of PMs, and thus leads to a large phase error. In CPMUs, this problem can be avoided by selecting the magnet temperature so that the remanent field reaches maximum and becomes less sensitive to temperature change. Nevertheless, the temperature gradient in CPMUs can cause a large phase error as explained in the following.

Let us recall the structure of an IVU as schematically shown in Fig. 1. The PM arrays are held by the in-vacuum beams usually made of aluminum that are supported by a number of in- and out-vacuum shafts. Then they are connected to the out-vacuum beams that are moved upward and downward to open and close the magnet gap. In the case of CPMUs, the out-vacuum beam is kept at room temperature, while the in-vacuum beam is cooled down to a cryogenic temperature. If the temperature gradient along the PM array is large, a nonnegligible gap variation is induced by difference in thermal shrink between the invacuum shafts.

In order to correct the phase error caused by the mechanism described above, we have to solve two problems: one is how to adjust the gap values at the in-vacuum beam positions, and the other is how to retrieve the information

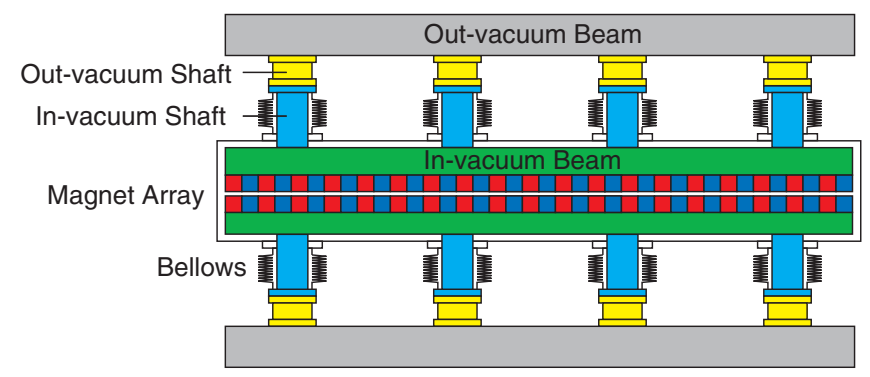

FIG. 1. (Color) Schematic illustration of the structure of an IVU. on the gap variation from the measured field distribution, which are explained in the following sections.

\section{A. How to adjust the gap values}

In order to adjust the gap value, we have developed a new out-vacuum shaft having a differential screw mechanism so that the total length can be adjusted. Because the out-vacuum beam is much more rigid than the in-vacuum beam, the gap value can be adjusted according to the length of the out-vacuum shaft, as is clear from Fig. 1.

The developed out-vacuum shaft has a similar structure to that of a turnbuckle as shown in Fig. 2 and is called the "differential adjuster." The difference from the turnbuckle is that the pitch distances of the two threads are slightly different (1.2 and $1.0 \mathrm{~mm}$ ), but the directions of the threads are identical. By revolving the middle part that corresponds to the metal loop of the turnbuckle, the distance $L$ can be controlled with a resolution of $0.2 \mathrm{~mm}$ per revolution. From our experience, the sensitivity of the gap correction is found to be better than $5 \mu \mathrm{m}$. The fixation screws are used to rigidly fix the position of the individual components after adjustment.

\section{B. How to retrieve the information}

The phase error, which specifies the undulator magnetic performance, is defined by

$$
\phi(z)=\frac{2 \pi \gamma^{2}}{\lambda_{u}\left(1+K^{2} / 2\right)} \int\left[\beta_{\perp}^{2}(z)-\left\langle\beta_{\perp}(z)^{2}\right\rangle\right] d z,
$$

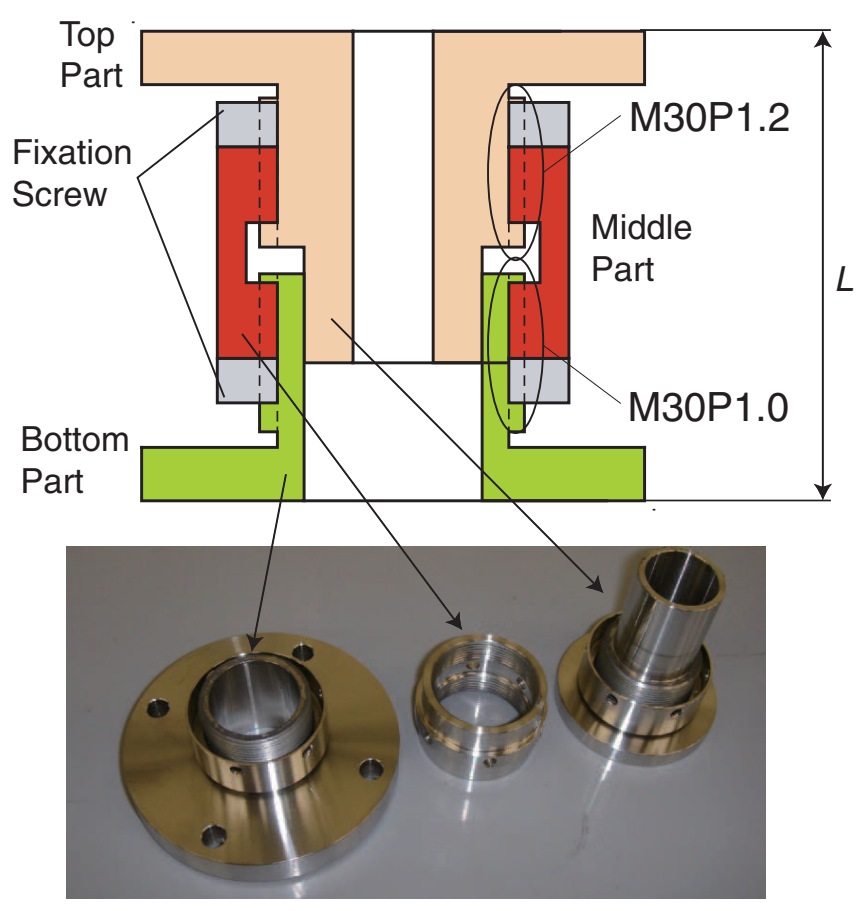

FIG. 2. (Color) Schematic illustration of the new out-vacuum shaft, or "differential adjuster." 
where $\lambda_{u}$ and $K$ are the undulator period and deflection parameter, $\gamma$ and $\beta_{\perp}$ are the Lorentz factor and transverse angle of the moving electron, and $\langle\cdots\rangle$ indicates an average over the undulator length. Note that a planar undulator is assumed in this section.

Let us now consider the CPMU field error that comes up at a cryogenic temperature. Assume that the field error is well corrected at room temperature, namely,

$$
\beta_{x} \simeq K \gamma^{-1} \cos \left(2 \pi z / \lambda_{u}\right), \quad \beta_{y} \simeq 0 .
$$

Because the field error after cooling the PM arrays is mainly due to the gap variation but not due to a variation in the magnetization vector of PMs, it is expected that the localized field errors, which give rise to unwanted trajectory wander or single kick errors, do not exist. Thus the resultant transverse angle has the form

$$
\beta_{\perp}(z)=K \gamma^{-1}[1+\eta(z)] \cos \left(2 \pi z / \lambda_{u}\right)
$$

where $\eta(z)$ is a slowly varying function of $z$ and can be regarded to be constant within one undulator period. To be specific, it denotes the peak field deviation from the nominal value.

Substituting Eq. (2) into Eq. (1) and averaging over one undulator period, we have

$$
\eta(z)=\frac{\lambda_{u}\left(1+K^{2} / 2\right)}{2 \pi K^{2}} \frac{d \phi(z)}{d z}
$$

We find that the field deviation function $\eta(z)$ is given by differentiating the phase error $\phi(z)$. Then it is easy to get the gap variation by calculating or measuring the dependence of the magnetic peak field on the magnet gap.

\section{MAGNETIC MEASUREMENT AT A CRYOGENIC TEMPERATURE}

In order to apply the field correction as explained in Sec. II, we need to measure precisely the magnetic field at a cryogenic temperature. For this purpose, a new system has been developed and installed in CPMU14.

The new system is based on the self-aligned field analyzer with laser instrumentation (SAFALI) system [6,7] developed for the measurement of IVUs for SPring-8 XFEL. The original SAFALI system was not applicable to the measurement under a ultrahigh vacuum (UHV) environment. In the new system, all the components have been modified to be compatible to UHV and thus it is called the in-vacuum SAFALI system. The schematic illustration of the measurement setup is shown in Fig. 3, and the crosssectional view along the $\mathrm{A}-\mathrm{A}^{\prime}$ line is shown in Fig. 4. As found from both the figures, the in-vacuum SAFALI system does not require a special vacuum chamber dedicated to measurement.

Instead of the massive granite bench that is usually used in the conventional field measurement system, a compact rail is inserted into the vacuum chamber and is supported by UHV-compatible two-axis linear stages composed of a couple of bellows to compensate the atmospheric pressure. The Hall sensor module is mounted on a carriage fitting to the rail and is moved along the undulator axis by means of a tensioned loop wire driven by a UHV-compatible stepper motor. Two irises with a diameter of $2 \mathrm{~mm}$ are attached to the Hall sensor module and two laser beams are introduced through the viewport to irradiate the irises and create two optical spots. During the movement along the undulator axis, the transverse position of the Hall sensor fluctuates due to a mechanical error and deflection of the rail. Such a positional error is detected by the position sensitive detectors (PSDs) as the fluctuation of the optical spot positions,

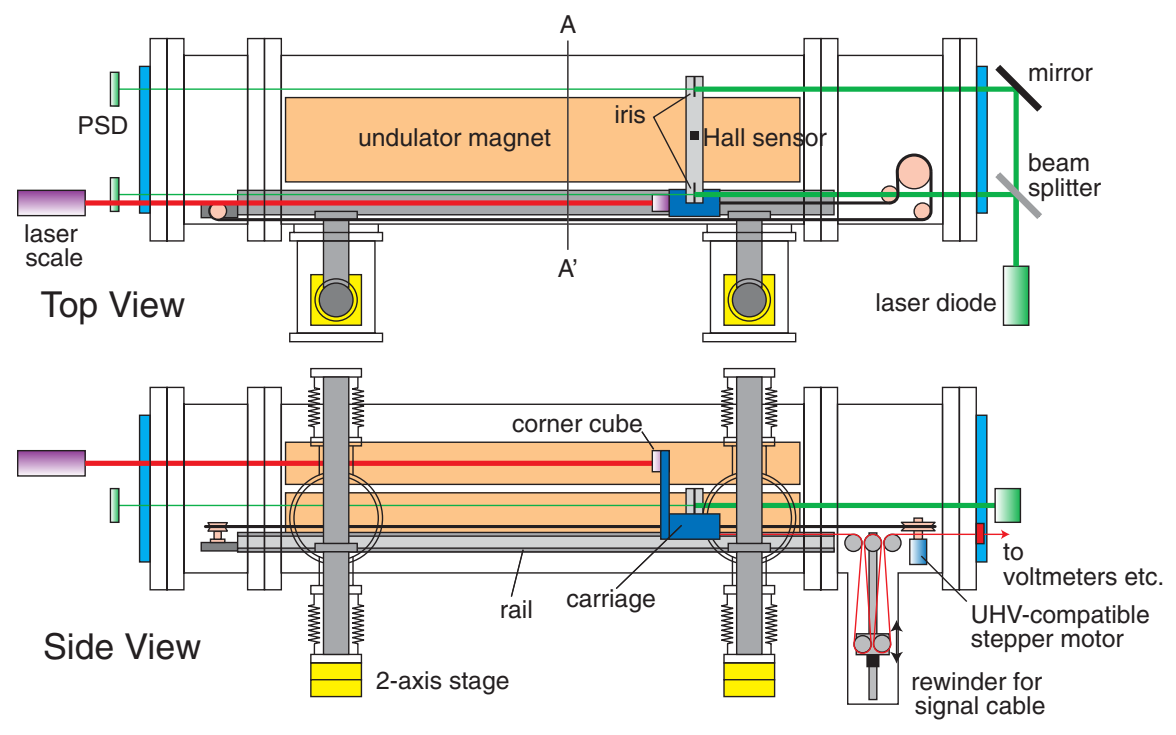

FIG. 3. (Color) Schematic illustration of the measurement setup. 


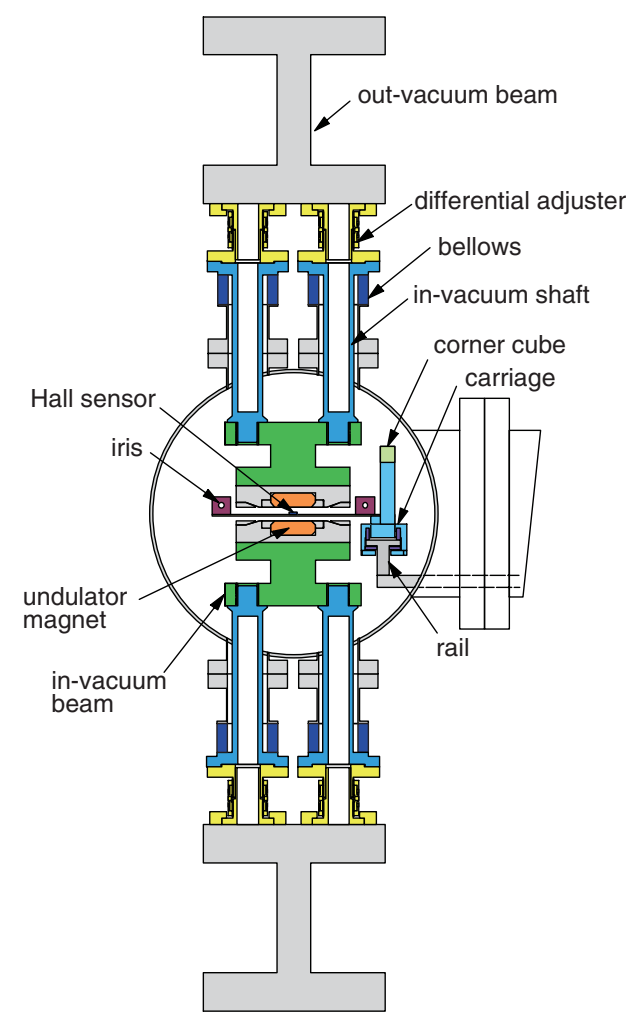

FIG. 4. (Color) Cross-sectional view taken along the line $\mathrm{A}-\mathrm{A}^{\prime}$ of Fig. 3.

and then corrected by translating the two-axis linear stages supporting the rail. The correction can be done in a feedback loop, or based on a feedforward table created in advance. In order to improve the accuracy, both methods can be used in combination.

The signal cable from the Hall sensor is connected to the vacuum feedthrough fixed on the vacuum flange. Because the Hall sensor is moved over the distance at least longer than the undulator length, we have to handle the long signal cable to avoid interference with other components inside the vacuum chamber, otherwise the cable can be damaged. This is not trivial because we do not have a lot of space for handling the cable as in the conventional measurement system placed in the atmosphere. In order to solve the problem, we have installed a cable rewinder composed of a movable pulley with weight and a UHV-compatible linear guide at one end of the vacuum chamber.

\section{RESULTS OF FIELD MEASUREMENT AND IN SITU CORRECTION}

After installing all the components for the field measurement described above, we measured the field distribution at the gap of $8 \mathrm{~mm}$ to check the magnetic performance at room temperature. The results are shown in red lines in Figs. 5(a) and 5(b) in terms of the 2nd field integral (beam trajectory) and phase error, respectively. The rms phase

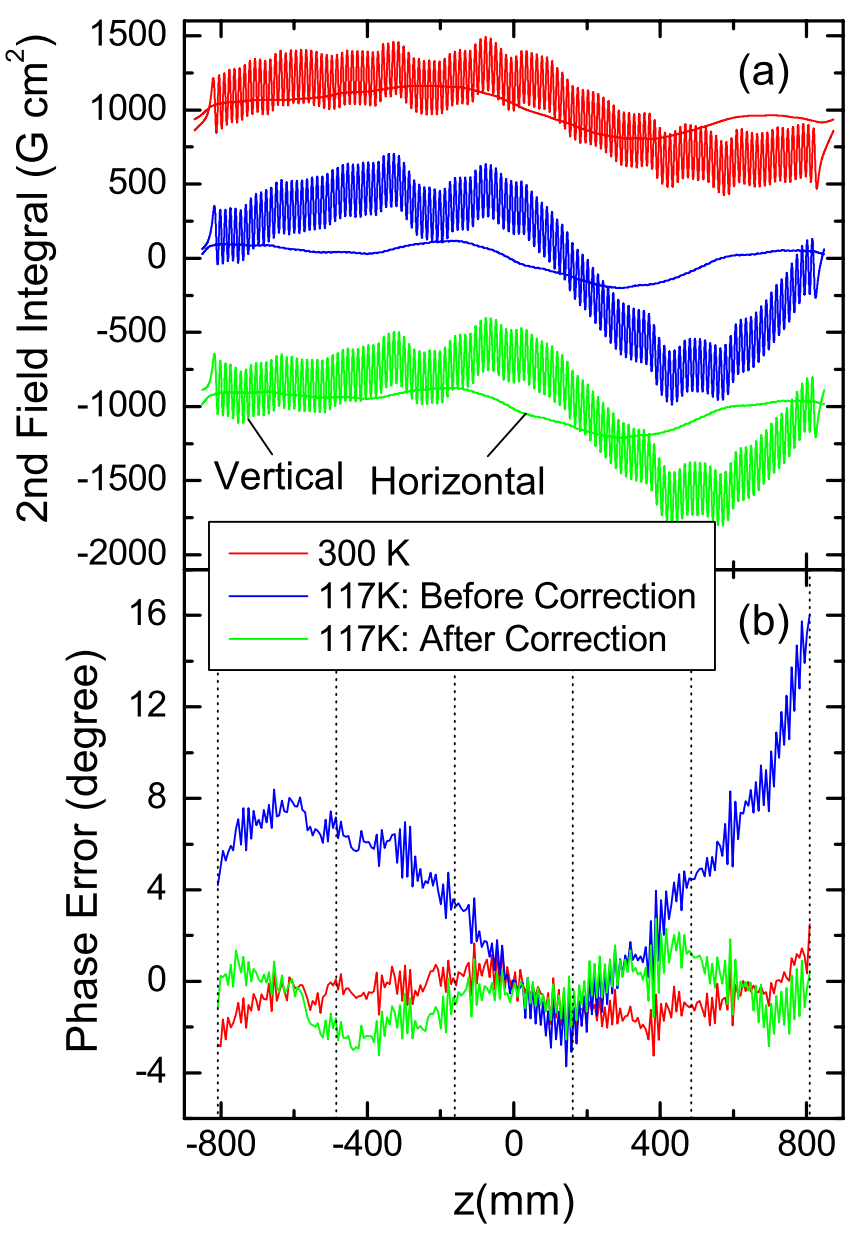

FIG. 5. (Color) Results of the field measurements in terms of (a) the beam trajectory and (b) the phase error. Dotted lines show the locations of the differential adjuster as the outer shafts. Note that the beam trajectories have been offset for readability.

error was found to be $1.0^{\circ}$, which was good enough to observe the performance degradation due to cooling.

We then started to cool down the PM arrays with the liquid nitrogen $\left(\mathrm{LN}_{2}\right)$ cooling system, which is originally dedicated to the monochromator cooling of SPring- 8 beam line. The lowest temperature of the PM arrays was $117 \mathrm{~K}$, at which the field measurement was carried out to compare with the measurement at room temperature. The results are shown in blue lines in Figs. 5(a) and 5(b), where we find that the rms phase error increased largely to $3.8^{\circ}$ as opposed to the small change of the beam trajectory. Obviously, this is attributable to the gap variation induced by temperature gradient. It is worth noting that the induced phase error is not correlated with the cooling geometry and thus the origin of the phase error is not clear.

In order to correct the phase error, we applied the in situ correction method described in Sec. II. First, we calculated the 6th order polynomial function by the least-square fitting of the phase error distribution function $\phi(z)$ and then differentiated it to obtain the field deviation function $\eta(z)$ 
according to Eq. (3). The reason why we have not directly differentiated $\phi(z)$ is to eliminate the rapidly oscillating term due to the trajectory error and retrieve the slowly varying function that corresponds to the phase error induced by temperature gradient. The order of polynomial was chosen to be equal to the number of in-vacuum shafts, i.e., the fixation points of the in-vacuum beam. For reference, the locations of the fixation points are indicated in dotted lines in Fig. 5(b). By analyzing $\eta(z)$, the approximate gap variations at these locations were found to be $-10 \mu \mathrm{m}, 0,+30 \mu \mathrm{m}, 0,-30 \mu \mathrm{m}$, and $-30 \mu \mathrm{m}$, respectively from left to right, which were corrected easily by the differential adjuster with the sensitivity better than $5 \mu \mathrm{m}$.

The result of correction is shown in green lines in Figs. 5(a) and 5(b). The rms phase error was reduced to $1.1^{\circ}$, comparable to that at room temperature. Note that the trajectory did not change before and after the correction, meaning that the trajectory error is not the main reason for the phase error increase due to cooling.

\section{SUMMARY}

We have shown that the phase error induced by temperature gradient in CPMUs can be corrected by means of the differential adjuster based on the precise field measurement with the in-vacuum SAFALI system and data analysis. Basically, the correction needs to be made only once before installation as far as the phase error induced by the cooling process is reproducible.

It should be stressed that this method is also applicable to normal IVUs to correct the phase error that is attributable to the slowly varying field deviation but not to the localized field errors. For example, let us consider two possible error sources to increase the phase error.

One is the reassembling of the PM arrays that is indispensable in the manufacturing process of IVUs. The field correction of IVUs is usually done without the vacuum chamber so that shimming or swapping the magnet units is possible. Then once after the PM arrays and in-vacuum shafts are removed from the out-vacuum shaft in order to assemble the vacuum chamber, they are installed again into the vacuum chamber. This reassembling process can give rise to the gap variation as in the case of the cooling process in CPMUs.

The other is the demagnetization of PMs due to the irradiation of the electron beam during a long-term accelerator operation. Even though the demagnetization level is not large compared to the remanent field of PMs, the phase error increase can be considerably large if the demagnetization is not uniform over the undulator length.

It is obvious that the field deviation caused by the above processes will probably be a slowly varying function, and thus the resultant phase error can be corrected by the in situ correction technique described in this paper.

\section{ACKNOWLEDGMENTS}

The authors thank Dr. M. Yamamoto and Dr. K. Hirata for their kind arrangement of the $\mathrm{LN}_{2}$ cooling system, and Dr. T. Takeuchi for his technical support during the cooling test.

[1] T. Hara, T. Tanaka, H. Kitamura, T. Bizen, T. Seike, T. Kohda, and Y. Matsuura, Phys. Rev. ST Accel. Beams 7, 050702 (2004).

[2] T. Tanabe, A. Blednykh, D. Harder, M. Lehecka, G. Rakowsky, and J. Skaritka, in Proceedings of the 21st Particle Accelerator Conference, Knoxville, 2005 (IEEE, Piscataway, NJ, 2005), p. 1949.

[3] T. Tanaka, T. Hara, T. Bizen, T. Seike, R. Tsuru, X. Marechal, H. Hirano, M. Morita, H. Teshima, S. Nariki, N. Sakai, I. Hirabayashi, M. Murakami, and H. Kitamura, New J. Phys. 8, 287 (2006).

[4] J. Chavanne, M. Hahn, R. Kersevan, C. Kitegi, C. Penel, and F. Revol, in Proceedings of the 11th European Particle Accelerator Conference, Genoa, 2008 (EPS-AG, Genoa, Italy, 2008), p. 2243.

[5] T. Tanaka, R. Tsuru, T. Nakajima, and H. Kitamura, J. Synchrotron Radiat. 14, 416 (2007).

[6] T. Tanaka, T. Seike, and H. Kitamura, in Proceedings of the 29th International Free Electron Laser Conference, Novosibirsk, 2007, p. 468.

[7] T. Tanaka, T. Seike, and H. Kitamura, Proceedings of the 30th International Free Electron Laser Conference, Gyeongju, 2008, p. 371. 